\title{
Utilisation des lipides non polaires dans les rouges à lèvres : état de l'art et perspectives
}

\author{
Entretien avec Zéphirin Mouloungui, \\ Carinne Alfos et Anne Rossignol-Castera
}

\author{
Zéphirin MOULOUNGUI ${ }^{1}$ \\ Carinne ALFOS ${ }^{2}$ \\ Anne ROSSIGNOL-CASTERA ${ }^{3}$ \\ ${ }^{1}$ Inra, Programme Chimie verte, \\ UMR 1010 - Chimie Agro-Industrielle INRA/ \\ INP-ENSIACET, Plate forme Lipotechnologique \\ INRA - CEPIA \\ 118 route de Narbonne - F-31077 TOULOUSE \\ <Zephirin.Mouloungui@ensiacet.fr> \\ ${ }^{2}$ Iterg, Département Lipochimie, \\ Rue gaspard Monge - Parc Industriel Bersol2, \\ F 33600 Pessac \\ $<$ c.alfos@iterg.com> \\ ${ }^{3}$ Iterg, Département Conseil \& Transfert, \\ Rue Gaspard Monge - Parc Industriel Bersol 2 \\ -F 33600 Pessac
}

\section{Pourquoi les lipides non polaires sont-ils les constituants lipidiques majeurs des rouges à lèvres?}

ZM : Selon Larson [1], les lipides non polaires ne forment pas de phases lipide-eau. Cependant, ils interagissent avec l'eau aux interfaces formant des monocouches à la surface de l'eau. Des matières premières domestiques aux produits élaborés directement utilisables, les lipides non polaires issus des végétaux ont conquis les cosmétiques notamment les rouges à lèvres. Ce sont des composants à la fois conventionnels, sophistiqués et évolutifs des formulations des rouges à lèvres. L'alternative naturelle est un des atouts de leur acceptabilité.

Les lipides non polaires dédiés à ces formulations sont répartis entre les huiles et matières grasses de commodité (soja, colza, palme, ricin, tournesol, palmiste, coprah, etc.), de spécialité (avocat, abricot, argane, germe de blé...), les beurres (karité, mangue, cacao, etc.) et les cires naturelles (montan wax, carnauba wax, candelilla wax...).

D'une façon générale, toutes ces matières premières sont des lipides non polaires constitués par des acides gras et leurs dérivés. De cette composition chimique se déclinent leurs fonctions essentielles, l'émollience et le pouvoir humectant. D'après Loubat-Boulenc, «l'émol-

\begin{abstract}
The nonpolar lipids represent a major fraction of the composition of the formulations of the lipsticks. Their functional role is elucidated. The functional approach offers reliable solutions to the complex problems concerned with these formulations by the means of combined technologies. It forecasts the innovation since the sourcing raw materials, along the chain of processes of extraction and transformation until working of the nonpolar lipids in the finished products.
\end{abstract}

Key words: lipsticks, lipids nonpolar, esters, protective waxes, emollience

lience décrit un ensemble de caractéristiques variables perçu à un moment donné par le toucher et la vue, qui évoque la douceur, l'élasticité et le pouvoir glissant pour le toucher, et le brillant et le mat pour la perception visuelle [2] ». Le film gras en surface, I'assouplissement de la peau, l'amélioration de l'élasticité relèvent de l'émollience, la réduction et/ou l'inhibition de la perte insensible de l'eau TEWL (Trans Epidermal Water Loss) est obtenu par le pouvoir humectant des lipides non polaires.

Outre le rôle basique de texturant, quelles sont leurs autres fonctionnalités?

ZM : La fonction est une approche dirigée vers la résolution d'un problème. Elle peut être définie comme la propriété de base à remplir pour répondre aux attentes du consommateur. Deux conditions sont nécessaires pour cette approche :

- une expertise pour définir les fonctionnalités désirées. Elle relève de la connaissance de la physico-chimie des constituants;

- un réseau de technologies fournissant ces fonctionnalités.

Les fonctionnalités sont identifiées : protection et effet barrière, conservation et/ou effets réparateurs, adhérence et effet de glissement, cohésion et effet de déformation, revêtement et effet rhéologique. Cette approche fonctionnelle impose une évolution de l'utilisation de la matière première. Du rôle basique d'ingrédients naturels, les lipides non polaires ont gagné un rôle d'ingrédients fonctionnels.

On assiste à une préférence accrue pour les ingrédients végétaux, natifs ou modifiés, au détriment des ingrédients exclusivement issus de synthèses chimiques comme les polymères.

Quels sont les nouveaux critères recherchés?

Ce phénomène est-il compatible avec le besoin d'ingrédients polyfonctionnels et synergiques?

ZM : Grâce à leur biodégradabilité, nontoxicité et non-écotoxicité, les esters complexes ou triacylglycérols des huiles de commodité ont contribué par exemple à déplacer les produits pétrochimiques non biodégradables comme les étoxylats toxiques. Biodégradabilité et écocompatibilité sont des nouvelles propriétés et des nouveaux critères exigés pour le futur des produits de beauté et de soins. Qui plus est, ces constituants majeurs des huiles et graisses naturelles jouent in situ le rôle de vecteur des lipides non polaires mineurs bioactifs que sont les tocophérols, phytostérols, tocotriénols... 
Ces constituants mineurs délivrent des fonctions anti-UV, antioxydants, conservateurs... Ces fonctionnalités exaltent ainsi le rôle $d^{\prime}$ 'ingrédients fonctionnels attribué aux huiles de spécialité et aux beurres. Source non conventionnelle de stéarine, le beurre de karité par exemple connaît un regain d'intérêt dans I'industrie des produits de soins grâce à la reconnaissance par l'expertise et celle des consommateurs de ses bienfaits thérapeutiques. Sa fonction humectante et ses propriétés régénératrices, anti-inflammatoires, anti-UV sont dues à la présence d'une fraction insaponifiable significative de $3-12 \%$ vecteur de plusieurs molécules bioactives: alcools triterpéniques, stérols, phénols, karitènes et hydrocarbures polyisopropéniques [3].

Parmi les huiles de commodité, I'huile de ricin s'est imposée par le caractère quasi homogène des acides gras des triacylglycérols la constituant. Son originalité réside dans le fait qu'elle possède une forte teneur en acide ricinoléique $(89,5 \%)$. Son indice d'hydroxyle est de 160 168. Grâce à ses groupes polaires hydroxylés, I'huile de ricin présente un indice de viscosité relativement élevé de 6,5-8. La présence d'une seule double liaison de configuration cis lui confère une très bonne fluidité, un haut pouvoir lubrifiant et un excellent pouvoir dispersant des colorants. Après déshydratation, I'huile de ricin devient siccative pour être utilisée en tant que base de vernis. C'est dans sa forme hydrogénée que l'huile de ricin se combine avec l'ensemble des cires, participe à I'industrie du revêtement. Les sels zinciques de ricinoléate manifestent des propriétés déodorante et désodorisante. Ces fonctionnalités sont intrinsèques aux structures moléculaires capables de chélater par le biais du groupe hydroxyle et du cation métallique les composés organiques responsables d'odeurs désagréables (dérivés oxygéné, azoté et soufré) inhibant ainsi leurs mauvaises odeurs [4]. Un effet déodorant est attribué aux ricinoléates de zinc dans les sprays, stick, poudre ou crème.

CA : Les formulations cosmétiques font appel à de nombreuses matières premières pour obtenir les performances techniques recherchées (tenue, couleur, résistance, brillance...) et les qualités cosmétiques (texture, confort, parfum, glissant...). En particulier dans le maquillage et les produits de soin, les produits étant en contact direct avec la peau, les qualités cosmétiques y prennent toute leur importance.

À ce titre, les polymères aussi bien pour les performances techniques que pour les qualités cosmétiques constituent des matières premières clés pour la cosmétique.

Cependant, les polymères sont souvent issus de la pétrochimie, synthétisés avec des procédés classiques et pas ou peu biodégradables. Leur substitution par des matières premières d'origine végétale permettrait de s'affranchir de la pétrochimie, de valoriser des agroressources et d'obtenir des matières premières dégradables.

Je pense que la recherche de synthons renouvelables, principalement issues du monde végétal, portant des fonctions susceptibles de réagir chimiquement pour la synthèse de nouveaux « biopolymères » selon un design à façon utilisant des procédés innovants est un axe porteur de développement.

Peut-on mesurer des effets synergiques entre certains lipides et les autres ingrédients des rouges à lèvres comme les pigments, les parfums et les filtres ? Comment créer une synergie ?

CA : Comme I'a explicité Hélène de ClermontGallerande: "La formulation n'est pas statique mais s'adapte continuellement aux exigences légales, toxicologiques, technologiques. Aujourd'hui, les silicones et esters ont remplacé les huiles végétales et minérales, les cires synthétiques, polyéthylène et polymères ont remplacé les cires végétales de carnauba et de candelilla; les rouges à lèvres proposés aujourd'hui ont bien évolué en quinze ans. » Ces différents constituants tels les esters d'acides gras, les huiles silicones et les polymères interagissent ensemble via des phénomènes de cocristallisation et d'interactions et permettent ainsi d'orienter la rhéologie afin d'obtenir des produits très fondants, brillants, confortables et de tenue correcte. Mais un retour au végétal, outre qu'il est dans l'air du temps, est par ailleurs possible. Certaines huiles végétales ou beurres natifs (beurre de kokum par exemple), huiles modifiées (non "grasses au toucher", présentant une bonne émollience et une bonne stabilité oxydative) et « biopolymères » pourraient remplacer avantageusement les silicones et les polymères d'origine minérale. L'étude de l'évolution du polymorphisme de ces différents mélanges, et du procédé de cristallisation pourrait déboucher sur la formulation de rouges à lèvres présentant une longue tenue et de bonnes qualités en termes de performances en revenant à un " sourcing » plus « végétal ».

On sait que les huiles végétales sont parfois sensibles à l'oxydation. La réponse à cette limite passe-t-elle forcément par la préconisation des cires dans une formule?

ZM : Un des intérêts majeurs des lipides nonpolaires est leur fonction protectrice contre les agressions environnementales associées à leur stabilité chimique et à la thermo-oxydation. Pour cette fonctionnalité, les esters complexes des triacylglycérols sensibles à l'oxydation de
I'air et à I'humidité sont supplantés par les esters d'acides gras et d'alkyles à longues chaînes. Les cires se sont imposées comme des candidats fiables de cette fonctionnalité. Sur la base de leur origine, les cires sont classées en cing groupes :

- cires minérales des ressources fossiles et pétrolières :

- cires animales de baleines, insectes...

- cires végétales de coton, riz, lin...

- cires synthétiques dérivés de polymères d'éthylène ou d'oxyde d'éthylène à longue chaîne ;

- autres composés assimilés de synthèse : les esters gras.

L'ensemble des constituants de ces groupes présente des molécules aux propriétés physiques similaires : points de fusion élevés, haut caractère d'hydrophobicité et haut degré de cristallinité (5). De ce fait, leur fonctionnalité s'exprime par des caractéristiques telles que l'adhésion et la cohésion aussi bien que le glissement et la déformation qui en font des agents de revêtement par excellence aux propriétés barrières avérées vis-à-vis de l'humidité et des radiations UV $[5,6]$.

Outre les cires solides, le monde végétal fournit des cires liquides. L'huile de jojoba est l'archétype des huiles de spécialité non glycéridique. C'est un mélange complexe dominé par des mono-esters de chaînes longues de 40 et 42 atomes de carbone composés d'acides gras et d'alcools gras de 20 et 22 atomes de carbone. Chaque élément hydrophobe porte une double liaison localisée entre le neuvième et le dixième atome de carbone à partir de la terminaison de la chaîne hydrocarbonée. La présence d'une insaturation de configuration cis dans les alcools gras et acides gras à longues chaînes les constituants confère à l'huile de jojoba un indice de viscosité de 225 [7]. Cette valeur est très élevée en comparaison avec les valeurs affichées par les esters encombrés totaux, mixtes ou complexes de néopentylpolyols largement utilisés dans la technologie des esters élaborés lubrifiants [8]. Avec un fort indice de viscosité, on peut avoir une forte viscosité à haute température tout en gardant une huile fluide à température ambiante ou à la température du corps. C'est un critère de stabilité vis-à-vis des contraintes de hautes températures.

En tant que substrat, l'huile de jojoba a été transformée en esters de jojoba par un processus d'interestérification [9]. L'élaboration des esters de jojoba avec conservation de la configuration cis est une clé de l'émollience apportée par le mélange d'esters composés de différents ratios de molécules présentant aucune, une ou deux doubles liaisons dans leurs chaînes hydrocarbonées. Les esters de jojoba démontrent une stabilité thermique 
remarquable évaluée par la mesure de l'indice de stabilité oxydative OSI (Oxidation Stability Index). Des valeurs OSI de 675 heures sont obtenues avec les esters de jojoba tandis que celles de l'huile de jojoba raffiné sont faibles, de I'ordre de 35 heures. Si bien que dans les applications de rouge à lèvres les esters de jojoba remplacent I'huile de ricin voire I'huile de jojoba. En plus de l'émollience et de la stabilité thermo-oxydative, ces esters améliorent l'étalement du stick avec réduction de la teneur des cires, tendent à promouvoir la dispersion uniforme des pigments et de la couleur.

Le mimétisme des fonctions et des propriétés des cires s'est répandu dans bon nombre de familles d'esters non polaires de synthèse. On peut citer le myristate d'isopropyle utilisé pour donner un film fin et homogène, le malate de diisostéraryle comme agent de brillance dans les rouges à lèvres. Les structures des esters encombrés constitués d'acides gras insaturés et alcools ramifiés confèrent la fluidité et tendent à améliorer l'étalement des émollients.

Ainsi les esters gras jouent un rôle fonctionnel majeur pour la qualité des produits de beauté et de soins pour le bien être des consommateurs $[2,10]$

CA : Prenons l'exemple des fonds de teints qui, il y a quelques années étaient formulés en émulsion huile dans eau avec une forte proportion d'huile végétale. Ce type de formulation permettait d'incorporer une quantité moindre d'huile végétale sensible à l'oxydation, mais le «touché » et la remontée en consistance n'étaient pas optimaux.

Aujourd'hui les fonds de teints sont formulés en émulsion inverse eau dans huile, ce qui leur confère un touché siliconé plus agréable et une bonne stabilité oxydative. Parmi leurs constituants, on retrouve les silicones et des hydrocarbures car ce « gras volatil » favorise l'étalement sur la peau et permet dans un second temps la remontée en consistance nécessaire pour le maintien sur la peau. Même si les triglycérides à chaînes moyennes sont de plus en plus utilisés en raison de la perception agréable liée aux chaînes courtes, les formulations à venir pourront recourir à des huiles végétales associant une stabilité à l'oxydation de part leur composition en acides gras (par exemple, les huiles oléiques telles que le tournesol oléique, le carthame oléique, I'huile de macadamia, huile d'amande douce ou encore de noyaux d'abricots), des teneurs élevées en antioxydants (telle l'huile d'olive riche en composés phénoliques), un touché non gras et de bonnes propriétés naturelles d'émollience (I'huile d'amandon de prune en est un bon exemple).

Les beurres végétaux répondent souvent à ces contraintes de stabilité à l'oxydation, notamment en émulsion, et présentent de bonnes propriétés d'émollience (beurres de cacao, d'illipe, de mangue, de kokum).

Enfin le « sourcing » offre la possibilité d'ores et déjà de recourir à de nouvelles sources végétales aux propriétés atypiques en raison de leur composition spécifique ; citons I'huile de ricin (bonne stabilité oxydative, huile dense, agent dispersant de pigments) et l'huile de tamanu (contenant des acides organiques non gras, riche en polyphénols et terpénoides). L'enjeu portera sur le contrôle de la traçabilité et de la variabilité de ces nouvelles matières premières en maîtrisant la filière sans oublier tous les développements d'huiles ou beurres modifiés à fonctionnalité spécifique, à partir de ces nouvelles sources, par procédé physique et/ou chimique.

L'huile de jojoba est-elle la seule cire naturelle disponible?

Les fleurs et les feuilles contiennent naturellement des cires protectrices.

Peut-on les exploiter à I'exemple de I'extrait lipidique de pollen de ciste?

AC À côté de la cire liquide de jojoba, il existe deux autres cires végétales largement connues et utilisées en cosmétique : la cire de carnauba et la cire de candelilla. La cire de carnauba, à haut point de fusion $\left(80-90^{\circ} \mathrm{C}\right)$ et riche en esters (80-85\%), est issue des feuilles du palmier Copernica cerifera. Cette cire assure un bon démoulage des sticks, amène dureté et consistance et donne de la brillance à la surface des produits cosmétiques. La cire de candelilla, moins riche en esters $(20 \%)$ mais riche en hydrocarbures (40-60\%), est issue d'un arbuste dénommé Euphorbia cerifera. Odorante du fait de la présence de terpènes et brillante, cette cire naturelle a un très bon pouvoir de rétention d'huile, améliore la stabilité et la texture des produits cosmétiques ainsi que leur toucher, leur tenue et leur finition. Les cires de carnauba et de candelilla sont souvent utilisées en association avec une cire d'abeille pour cou- pler leurs fonctionnalités respectives et donner une texture plus ou moins souple au produit fini. L'extrait lipidique de pollen de ciste est quant à lui un bon exemple de produit innovant. II contient des esters vrais et des triglycérides d'acides gras mais aussi de nombreux composés insaponifiables (stérols, caroténoïdes, tocophérols) et des polyphénols. Ces composés lui conférent à la fois une texture type « miel » et une émollience intéressantes associées à un pouvoir hydratant et anti-radicalaire. Cependant, le procédé d'extraction est long et complexe et le développement industriel de l'extrait de pollen de fleur passe par une optimisation des coûts de production. Ce verrou est du reste aussi celui qui pourrait limiter le développement de cires de fleurs ou de feuilles car les quantités présentes et potentiellement valorisables sont généralement faibles.

\section{RÉFÉRENCES}

1. LARSSON K. Basic concepts. In : Lipids molecular organization, physical fonctions and technical application. The oily press LTD, 1994 : 1-4; (Vol. 5, chap. 1).

2. LOUBAT BOULENC N. Esters en cosmétologie : généralités et fonctionnalités. OCL 2004 . $11(6)$ : 454-6.

3. LOVETT PN. Shea butter industry expanding in west Africa. Inform 2005 ; 16(5) : 273-5.

4. KUBN H, MULLER F, PEGGAN I, ZEKERN R. Mechanism of the odor adsorption effect of zinc ricinoleate. A molecular dynamics computer simulation. / Surfact Deterg 2000 ; 3(3) : 335-43.

5. MATTHIES L. Natural montan wax and its raffinates. Eur J Lipid Sci Technol 2001 ; 103 : 239. 48.

6. SHELLHAMMER TH, KROCHTA JM. Edible coatings and film barriers. In: Gunstone FD, Padley FB, eds. Lipids Technologies and Applications. Marcel Dekker Inc. chap. 17, 1997 : 453 79.

7. WISMAK J. In : Jojoba Oil. The chemistry and technology of jojoba oil. AOCS. Chap 1, 1987 : 1-71.

8. EYCHENNE V, MOULOUNGUI Z. Relationships between structure of neopentylpolyols esters. Ind Eng Chem Res 1998 ; 37 : 4835-43.

9. KLEIMAN R, DWYER K. A natural alternative. Inform 2006 ; 17(6) : 346-7.

10. MEFFERT A. Technical uses of fatty acid esters. J Am Oil Chem Soc 1984 ; 61(2) : 255-8. 